\title{
THE JUNE MEETING IN PULLMAN
}

The four hundred seventy-first meeting of the American Mathematical Society was held at the State College of Washington, Pullman, Washington, on Saturday, June 16, 1951, following the meeting on Friday of the Pacific Northwest Section of the Mathematical Association of America.

Approximately 65 persons attended the meetings including the following 43 members of the Society:

H. A. Antosiewicz, T. M. Apostol, R. W. Ball, C. R. Ballantine, J. P. Ballantine, R. A. Beaumont, J. S. Biggerstaff, Z. W. Birnbaum, J. L. Bottsford, J. L. Brenner, L. G. Butler, Harold Chatland, P. A. Clement, K. L. Cooke, C. M. Cramlet, D. B. Dekker, W. F. Donoghue, A. L. Duquette, Trevor Evans, R. E. Fullerton, K. S. Ghent, J. W. Green, F. L. Griffin, M. E. Haller, A. E. Halteman, I. M. Hostetter, R. D. James, J. M. Kingston, M. S. Knebelman, R. B. Leipnik, J. J. Livers, A. T. Lonseth, R. E. Lowney, L. H. McFarlan, H. B. Mann, A. F. Moursund, D. C. Murdoch, Ivan Niven, L. J. Paige, M. F. Ruchte, J. R. Vatnsdal, R. M. Winger, Fumio Yagi.

The meeting opened Saturday morning with a session for contributed papers, Professor M. S. Knebelman presiding. Following this was the invited address, The asymptotic density of sequences, by Professor Ivan Niven, of the University of Oregon. Professor R. D. James presided at the invited address.

On Friday evening there was a joint dinner for the Society and Association, followed by a social hour in the Library. On Saturday afternoon, those attending were entertained for tea at the home of Professor Knebelman.

Following the invited address; a resolution was passed to request the Council of the Society to schedule a meeting in Eugene, Oregon in June, 1952, in conjunction with the June meeting there of the Pacific Northwest Section of the Mathematical Association of America, and in connection with celebration of the seventy-fifth anniversary of the founding of the University of Oregon.

Abstracts of papers presented at the meeting follow. Papers whose titles are followed by $t$ were presented by title. Mr. McGregor's paper was presented by Professor R. D. James. Mr. Kiefer was introduced by Professor Jacob Wolfowitz, Mr. Liao by Professor E. H. Spanier.

\section{Algebra and Theory of Numbers}

\section{9t. T. A. Botts: Representation of algebras by filters.}

A very simple construction by abstract filters, equivalent to a construction of Dunford's [Nelson Dunford and M. H. Stone, On the representation theorem for Boolean algebras, Revista de Ciencias (Lima) vol. 43 (1941) pp. 447-453] by well-ordering, 
is given for a homomorphic point-set representation of any commutative, associative, and idempotent multiplicative system with zero, the construction being made so as to yield classical representation theorems for distributive lattices and for Boolean rings. (Received April 23, 1951.)

\section{J. L. Brenner: The problem of unitary equivalence.}

In this article, a canonical form is defined for an arbitrary (singular or nonsingular) $n^{2}$ matrix $A_{1}$ over the complex numbers, under unitary transformation. The group of matrices which fix the canonical form is found, and more generally a canonical form is defined for any matrix under transformation by any such group. This gives an effective method for considering the simultaneous equations $X A_{i} X^{*}=B_{i}, X X^{*}=1$ $(i=1,2, \cdots)$. The steps in the inductive definition of a canonical form involve either rational operations or the finding of characteristic roots of matrices of order $n$ or less. The first step in the analysis is to transform $A_{1}$ so that $A_{1} A_{1}^{*}$ is diagonal. This article will be published in Acta Mathematica. (Received April 30, 1951.)

\section{1t. D. R. Fulkerson: Hermite forms of row-finite matrices.}

Let $\mathfrak{M}$ denote the ring of row-finite matrices over a field $F$. If $A \in \mathfrak{M}$, there exists a nonsingular $P \in \mathfrak{M}$ such that $P A=H$ has the following form: the nonzero rows $\phi_{i}$ of $H$ have lengths $l_{i}$ with $l_{i}<l_{i+1} ; \phi_{i}$ has 1 in the $l_{i}$ position, zeros in the positions $l_{1}, \cdots, l_{i-1}$. The matrix $H$ is said to be an Hermite form of $A$. Two Hermite forms are left associates if and only if they differ by a permutation of rows, and consequently arbitrary $A, B \in \mathfrak{M}$ are left associates if and only if they have the same row space and nullity. From an Hermite form of $A$ one readily deduces a theorem due to Toeplitz: $A$ is equivalent to $T$ where each row of $T$ has at most one 1 , zeros elsewhere, and the nonzero rows have increasing lengths. $T$ is referred to as a Toeplitz form of $A$. Two Toeplitz forms are equivalent if and only if they differ by a permutation of rows and columns, whence $A$ and $B$ are equivalent if and only if they have the same rank, nullity, and defect. The reduction to Toeplitz form is used to show that $\mathfrak{M}$ is regular and has only one proper two-sided ideal. Finally, row-finite linear systems of equations are treated by reducing the matrix of coefficients to Hermite form. (Received April 26, 1951.)

\section{2t. R. A. Macauley: Polynomial valuations.}

If $R$ is a field on which all (nonarchimedean) valuations are known, then all valuations on $R[x]$, where $x$ is transcendental over $R$, are also known. Ostrowski described such valuations of $R[x]$ by means of pseudo-convergent sequences in the algebraic completion $A$ of $R$. MacLane showed that if all valuations of $R$ are discrete, then any valuation $V$ of $R[x]$ can be represented by certain "key" polynomials in $R[x]$. The present paper exhibits the connection between these two treatments. This is achieved by first determining keys for the valuation which a pseudo-convergent sequence defines on $A[x]$, and then relating these keys to those for $V$. (Received May 1, 1951.)

403. H. B. Mann: On the number of integers in the sum of two sets of positive integers.

Let $\alpha$ be the density of the set $A, \bar{\alpha}$ its asymptotic density, $\alpha^{*}$ its Besicovitch density, and $\alpha_{1}$ its modified density as defined by Erdös (Ann. of Math. vol. 43 (1942) pp. 65-68). Let further $A(n)$ denote the number of positive integers in $A$ that do not 
exceed $n$. It is proved if $A+B=C$ and if $n$ is not in $C$ then (1) $C(n) \geqq \alpha_{1} n+B(n)$. This is an improvement over a previous result of the author (Ann. of Math. vol. 43 (1942) pp. 523-527) according to which $C(n) \geqq \alpha^{*} n+B(n)$. Equation (1) immediately yields: $\bar{\gamma} \geqq \alpha_{1}+\bar{\beta}$. The latter inequality is sometimes better and sometimes not as good as Erdös' $\bar{\gamma} \geqq \bar{\alpha}+\bar{\beta} / 2$, which however has been established only if there exists a number $b \subset B$ such that $b+1 \subset B$. (Received May 4,1951 .)

\section{4t. M. A. Melvin: Metric classification of the bounded affinity} groups.

Consider all groups of bounded affine transformations about a fixed point in a real or complex euclidean space of $n$ dimensions ( $n$ finite or infinite). Both finite and infinite orders occur. For many geometric and physical purposes (see, for instance, the point of view developed in Symmetry and affinity of electromagnetic fields charges and poles, M. Avramy Melvin, Proceedings of the Canadian Mathematical Congress 1949 (in press); there, however, most of the groups involved are unbounded, and will be discussed in a subsequent paper), the usual replacement of any such bounded affinity group $(A)$ by its affinely equivalent unitary group $(U)$ is not desirable. This is because such a transformation effaces the dilatometric properties of the affine group, replacing the component strain ("ellipsoid" or Hermitian matrix) associated with each affine transformation by the identity. Thus we propose a further classification of affinity groups in which $T^{-1}(A) T$ is considered equivalent to $(A)$ only when $T$ is unitary. This determines the metric class to which $(A)$ belongs, and which is included with other metric classes in the broader affine class represented by $(U)$. A general prescription will be given, and applied, for the construction of the metric classes associated with any given affine class. Except for the trivial case $n=1$, there are infinitely many such metric classes. However, for a finite-dimensional group the number of "degrees of freedom" of this infinity, or the metric multiplicity of the affine class, is a finite integer. (Received May 2, 1951.)

\section{L. J. Paige: Simultaneous reduction of matrices to triangular form.}

The author proves: A necessary and sufficient condition for two matrices $A$ and $B$ to have an eigenvector in common is that $A$ and $B$ commute on an invariant subspace $L$ of $A$ and $B$. A simple inductive proof of Frobenius' theorem regarding the eigenvalues of $f(A, B)$ is then given. The same method may be used to obtain a result of McCoy (Bull. Amer. Math. Soc. (1936) p. 593). (Received May 3, 1951.)

406t. G. N. Raney: Completely distributive complete lattices. Preliminary report.

If $L$ is a complete lattice of which it is assumed only that meets are completely distributive with respect to joins, then the mapping which takes every $M$-closed subset of $L$ into its join is a complete-homomorphism of the lattice of $M$-closed subsets of $L$ onto $L$. This is based on the fact that in a complete lattice the intersection of any family of $M$-closed subsets is the same as the set of meets of selections over the family. Furthermore, the family of $M$-closed subsets of any lattice is a complete ring of sets. The lattice of a complete ring of sets is complete and completely distribuive, and the image of a completely distributive complete lattice under a completehomomorphism is a completely distributive complete lattice. Therefore, $L$ is completely distributive and, in particular, joins are completely distributive with respect 
to meets in $L$. In this manner it is shown that either condition for complete distributivity implies the dual condition, and that a lattice is complete and completely distributive if and only if it is a complete-homomorphic image of a complete ring of sets. (Received April 19, 1951.)

\section{ANALYsis}

407. T. M. Apostol: On the Lerch zeta function.

The function $\phi(x, a, s)=\sum_{n=0}^{\infty} \exp (2 n \pi i x)(n+a)^{-s}$ satisfies $\phi(x, a, 1-s)$ $=(2 \pi)^{-s} \Gamma(s) \exp (-2 \pi i a x)\{\exp (\pi s i / 2) \phi(-a, x, s)+\exp (2 a \pi i-\pi s i / 2) \phi(a, 1-x, s)\}$ which was first proved by Lerch using contour integration as in Riemann's first proof of the functional equation for $\zeta(s)=\phi(0,1, s)$. Riemann's second method, based on the transformation theory of theta functions, does not yield Lerch's relation as might be expected but leads instead to the formula $\Lambda(x, a, 1-s)=2(2 \pi)^{-s} \cos (\pi s / 2) \Gamma(s)$ $\cdot \exp (-2 \pi i a x) \Lambda(-a, x, s)$ where $\Lambda(x, a, s)=\phi(x, a, s)+\exp (-2 \pi i x) \phi(-x, 1-a, s)$. Lerch's relation is derived from this using, in addition, the differential-difference equations for $\phi$. The paper also contains two methods for evaluating $\phi(x, a, s)$ for negative integral values of $s$ as finite sums of elementary functions. The results are analogous to the evaluation of the Hurwitz zeta function $\zeta(s, a)=\phi(0, a, s)$ in terms of Bernoulli polynomials for $s=0,-1,-2, \cdots$. (Received April 24, 1951.)

\section{8t. T. M. Apostol: Remark on the Hurwitz zeta function.}

Although the Hurwitz zeta function $\zeta(s, a)$ is obtained from the Lerch zeta function $\phi(x, a, s)$ by putting $x=0$ (see Bull. Amer. Math. Soc. Abstract 57-5-407 for definitions), the functional equation for $\zeta(s, a)$ cannot be so obtained from that of $\phi$. In this paper the author proves that $k^{1-s} \zeta(s, a / k)-\zeta(s, a)=\sum_{t=1}^{k-1} \phi(t / k, a, s)$ for any positive integer $k$ and uses this to derive Hurwitz's relation $\zeta(1-s, a)$ $=2 \Gamma(s)(2 \pi)^{-s} \sum_{n=1}^{\infty} \cos (\pi s / 2-2 \pi a n) n^{-s}$ from Lerch's functional equation for $\phi$. The proof requires a knowledge of the behavior of $\zeta(s, a)$ for $a \rightarrow 0$ in certain regions of the s-plane. (Received April 24, 1951.)

409t. Bent Fuglede and R. V. Kadison: On a conjecture of Murray and von Neumann.

A ring of operators $M$ (that is, a weakly closed self-adjoint algebra of operators) is said to be "normal" when each subring of $M$ is the set of all operators in $M$ which commute with the set of operators in $M$ which commute with the given subring (cf. F. J. Murray and J. von Neumann, On rings of operators, Ann. of Math. vol. 37 (1936) pp. 116-229). Murray and von Neumann prove that all factors of type I are normal and exhibit examples of factors of type II which are non-normal. They conjecture that all factors of type II are non-normal. This conjecture is proved in the present note, and, in fact, it is shown that one can produce a subfactor of the given factor which violates the normalcy. (Received May 2,1951.)

\section{J. W. Green: On an expansion method for parabolic partial differential equations.}

Let $L(u) \equiv u_{t}-a u_{x x}-b u_{x}-c u$, where $a, b$, and $c$ are functions of $x$ and $t$. The problem considered is to solve $L(u)=F(x, t)$, in the region $t>0,0<x<\pi$, subject to the condition that $u$ vanish on the boundary. The method of solution is an adaptation of a method developed by S. E. Faedo (Publicazioni dell' Istituto per le Applicazioni del Calcolo, no. 247, Rome, 1949) for the solution of hyperbolic equations. An approxi- 
mation is determined in the form $u_{n}=\sum_{j=1}^{n} c_{n, j}(t) \sin j x$ where the $c_{n, j}(t)$ are determined by the system of ordinary differential equations, $\int_{0}^{\pi} L\left(u_{n}\right) \sin j x d x=0, j=1,2, \cdots, n$. By employing partial integration in a manner analogous to that in which uniqueness is usually proved, bounds are obtained for the integrals of the square of $u$ and its various derivatives, permitting convergence of the $u_{n}$ to a solution $u$ to be shown. Estimates for $u-u_{n}$ and $\int_{0}^{\pi}\left(u-u_{n}\right)^{2} d x$ are obtained. (Received May 2, 1951.)

411. A. T. Lonseth: On the error incurred in solving nonhomogeneous linear functional equations by Galerkin's method.

Suppose that an equation $L u=f$ is to be solved, where $L$ is linear, and that $u$ must also satisfy certain conditions $B$ on the boundary of its region $R$ of definition. Let $\phi_{0}$ satisfy $B$, and let $\phi_{1}, \cdots, \phi_{n}$ be orthonormal on $R$ and satisfy the homogeneous boundary conditions corresponding to $B$. Then solution $u$ is approximated by a linear combination $u_{n}$ of the $\phi$ 's, with coefficients determined so that $L u_{n}-f$ is orthogonal to each coordinate function $\phi_{1}, \cdots, \phi_{n}$ on $R$. With quadratic norm, $\left\|u-u_{n}\right\|$ is bounded above if $L$ has a bounded inverse; bounded below if $L$ is bounded. Applications are made to classes of differential and integral equations. (Received May 6, 1951.)

\section{J. L. McGregor: An integral of Perron type.}

In the definition of the Perron integral of a function $f(x)$ over a closed interval $[a, b]$ a major function $M(x)$ and a minor function $m(x)$ are required to satisfy the conditions (i) $M(x)$ and $m(x)$ are continuous on $[a, b]$ and $M(a)=m(a)=0$; (ii) $-\infty \neq D_{*} M(x) \geqq f(x) \geqq D^{*} m(x) \neq+\infty$. It is shown that without restricting the generality of the integral one may impose the additional condition (iii) $M(x)$ and $m(x)$ are differentiable on $[a, b]$. (Received May 1, 1951.)

413t. E. J. McShane: Order-preserving maps and integration processes.

Daniell's method of defining the Lebesgue integral is to start with an elementary integral, which is an order-preserving map of a subset of a lattice of functions into the real number system satisfying a certain set of postulates, and by devices based primarily on order properties to extend the domain of definition of the elementary integral so as to obtain a mapping with the well known continuity and closure properties possessed by the Lebesgue integral. By methods resembling those of Daniell, it is shown that an order-preserving map of a subset of a lattice $F$ into a partially ordered set $G$ can, under suitable assumptions, be extended so as to obtain a mapping of a larger subset of $F$ into $G$ having continuity and closure properties like those of the Lebesgue integral. As a preliminary, it is necessary to devise suitable definitions of closure, completeness, and convergence in partially ordered sets. Even when $F$ is a lattice of functions and $G$ the system of real numbers the present postulates are less restrictive than those of Daniell, so that the special cases include non-absolutelyconvergent integrals as well as the Lebesgue-Stieltjes integral. By choosing $G$ to be the set of bounded hermitian operators on a Hilbert space, one obtains the spectral resolution of such operators, and of bounded self-adjoint algebras of linear operators. (Received May 1, 1951.)

414. George Marsaglia: On the compatibility of distribution functions.

Consider an index set $T$ and elements $x_{t}, t \in T$. Let the integer $k$ be fixed, and 
suppose that for every subset $t_{1}, \cdots, t_{n}$ of $T$, with $n \leqq k$, there is assigned a distribution function $F_{x_{t_{1}}} \cdots, x_{t_{n}}\left(a_{1}, \cdots, a_{n}\right)=P\left[x_{t_{1}} \leqq a_{1}, \cdots, x_{t_{n}} \leqq a_{n}\right]$. Can such a system be extended to a stochastic process? Yes. For each $t \in T$, let $P_{t}$ be the semi-ring of half-open intervals (possibly infinite) of $R_{1}$, the reals. Let $\mathcal{X} C$ be the class of cartesian products $\prod_{t \in T} M_{t}$, where $M_{t} \in P_{t}$ and $M_{t} \neq R_{1}$ for at most a finite number of $t$ 's; let the number of exceptions to $M_{t}=R_{1}$ be the index of a set in $\mathcal{X}$, and let $\mathcal{X} \mathcal{C}_{k}$ be the sets of $\mathcal{X}$ having index not greater than $k$. The principal result is to the effect that such a system of distribution functions determines a set function on $\mathcal{X}_{k}$ which may be extended to $\mathcal{X}_{k+1}$ and hence to the semi-ring $\mathcal{X}$. Well known methods then yield a measure which determines the stochastic process. (Received April 30, 1951.)

\section{5t. K. S. Miller: The one-sided Green's function.}

A function $H(x, \zeta)$ called the one-sided Green's function is defined. It bears the same relation to Volterra type integral equations as the classical Green's function $G(x, \zeta)$ does to Fredholm integral equations. Various properties of $H(x, \zeta)$ are deduced. For example, if $H(x, \zeta)$ is the Green's function for the linear differential operator $L$, then a fundamental system of solutions can be constructed in terms of $H$ and its derivatives evaluated at the initial point. This function, which is closely related to the impulsive response of linear systems, is of use in various physical problems (particularly network theory) where interest is centered on one boundary point only. (Received May 9, 1951.)

\section{6t. L. B. Robinson: A complete system of tensors. II.}

The author computes a complete system of tensors for the case $\langle r=2\rangle$ associated with the system (A) $y_{1}^{\prime \prime}+p_{11} y_{1}^{\prime}+p y_{2}^{\prime}+q_{11} y_{1}+q y_{2}, y_{2}^{\prime \prime}+p y_{1}^{\prime}+p_{22} y_{2}^{\prime}+q y_{1}+q_{22} y_{2}$. Write $V_{11} \equiv \Theta^{1 / 2}\left\{y_{1}^{2} I_{22}-2 y_{1} y_{2} I_{12}+y_{2}^{2} I_{11}\right\}=F_{11}, \quad V_{12} \equiv \Theta^{1 / 4}\left\{y_{1} Y_{1} I_{22}-\left(y_{1} Y_{2}+y_{2} Y_{1}\right) I_{12}+y_{2} Y_{2} I_{11}\right\}$ $=F_{12}, \quad V_{22} \equiv\left\{Y_{1}^{2} I_{22}-2 Y_{1} Y_{2} I_{12}+Y_{2}^{2} I_{11}\right\}=F_{22}, \quad V_{13} \equiv D\left\{y_{1} I_{23}-y_{2} I_{13}\right\}=F_{13}, V_{23} \equiv \Theta^{-1 / 4} D$ - $\left\{Y_{1} I_{23}-Y_{2} I_{13}\right\}=F_{23}$, and $V_{33} \equiv \Theta^{-1 / 2} D^{2} I_{33}=F_{33}$. The $F^{\prime}$ 's are arbitrary functions of the covariants which have been long known. If the $V$ 's are solved with respect to the $I$ 's, the complete system of tensors results. (Received April 23, 1951.)

\section{Statistics and Probability}

\section{7t. J. C. Kiefer: On Wald's complete class theorems.}

Terminology of A. Wald (Statistical decision functions, New York, Wiley, 1950) is used in what follows. Let $\mathcal{D}$ be the class of all decision functions available to the statistician, let $\mathcal{D}_{b}$ be the class of all decision functions with bounded risk functions, let $C_{w}$ be the class of all Bayes solutions in the wide sense, and let $C_{s}$ be the class of all Bayes solutions in the strict sense. Wald showed that under assumptions 3.1 to 3.6 of the above reference, $C_{w}$ is complete relative to $\mathcal{D}_{b}$ (Theorem 3.17); and that under assumptions 3.1 to $3.7, C_{s}$ is complete relative to $\mathcal{D}_{b}$ (Theorem 3.20). (The same results were also proved by Wald in previous publications under stronger assumptions.) The present author proves that the above theorems remains true if in their statement $\mathcal{D}_{b}$ is replaced by $\mathcal{D}$. Under suitable assumptions, the method of proof is applicable to games whose payoff functions are bounded from below but not from above. (Received May 23, 1951.)

\section{TOPOLOGY}

418t. S. T. Hu: Cohomology rings of compact connected groups and their homogeneous spaces. I. 
It is a classical result of Elie Cartan that the cohomology ring $H(X)$ with real coefficients of a homogeneous space $X=G / G_{*}$ of a compact connected Lie group $G$ over a connected subgroup $G_{*}$ is a local property, namely, it depends only on the Lie algebra $L$ of $G$ and the subalgebra $L_{*}$ of $L$ corresponding to $G_{*}$. In the present paper, this theorem is generalized to the compact connected topological groups as follows. Let a compact connected topological $G$ operate transitively on a Hausdorff space $X$ and let $G_{*}$ denote the subgroup of $G$ consisting of the elements of $G$ which leave a given point $x_{*}$ fixed. Then the Alexander cohomology ring $H(X)$ with real coefficients of the homogeneous space $X$ depends only on the points of any given open neighborhood $U$ of $x_{*}$ in $X$ and the operations on the points of $U$ determined by the elements of $G_{*}$ and those of any given open neighborhood $V$ of the neutral element $e$ in $G$. (Received April 30, 1951.)

\section{9t. S. T. Hu: Cohomology rings of compact connected groups and their homogeneous spaces. II.}

Let $V$ be a given local group and $V_{*}$ a subgroup of $V$. A cohomology ring $H\left(V, V_{*}\right)$ with real coefficients is defined as follows. For $p \rightarrow 0$, a $p$-function of $V \bmod V_{*}$ is a continuous real function $\phi: W^{p} \rightarrow R$ defined on $W^{p} \subset V^{p}$ for some open neighborhood $W$ of $e$ in $V$ such that $\phi\left(g v_{1} h_{1}, \cdots, g v_{p} h_{p}\right)=\phi\left(v_{1}, \cdots, v_{p}\right)$ for all $v_{i} \in W, g \in V_{*}$, $h_{i} \in V_{*}$ provided that $g v_{i} h_{i} \in W(i=1, \cdots, p)$. Two $p$-functions $\phi$ and $\psi$ are said to be equivalent if they are equal for the arguments sufficiently near $e$. Then the $p$-functions are thus divided into equivalence classes called the $p$-cochains, $C^{p}$. Put $C^{0}=R$ and $C=\sum_{p} C^{p}$. $C$ forms a graded ring with coboundary operator $\delta: C \rightarrow C$ as follows. For any $p$-function $\phi$ and any $q$-function $\psi$, define $\left(\phi \bigcup_{-1} \psi\right)\left(v_{1}, \cdots, v_{p+q}\right)$ $=\phi\left(v_{1}, \cdots, v_{p}\right) \psi\left(v_{p}^{-1} v_{p+1}, \cdots, v_{p}^{-1} v_{p+q}\right), \quad(\delta \phi)\left(v_{1}, \cdots, v_{p+1}\right)=\phi\left(v_{1}^{-1} v_{2}, \cdots, v_{1}^{-1} v_{p+1}\right)$ $+\sum_{i=1}^{p+1}(-1)^{i} \phi\left(v_{1}, \cdots, \hat{v}_{i}, \cdots, v_{p+1}\right)$ for $v_{i}$ in a sufficiently small open neighborhood of $e$ in $G$. Then $H\left(V, V_{*}\right)$ is the cohomology ring of the graded ring $C$. Now let $X=G / G_{*}$ be the homogeneous space of a compact connected group $G$ over a connected subgroup $G_{*}$. Let $V$ be any open neighborhood of $e$ in $G$ and $V_{*}=V \cap G_{*}$. It is proved in this paper that the Alexander cohomology ring $H(X)$ with real coefficients is isomorphic with the ring $H\left(V, V_{*}\right)$. (Received April 30,1951.)

\section{0t. S. D. Liao: On periodic transformations of homology spheres.}

Let $Q$ be a compact Hausdorff space of finite dimension and $T$ a periodic transformation of prime period $p$ operating on $Q$. It was shown by Smith that if $Q$ is a homology $n_{0}$-sphere over the group $G_{p}$ of integers modulo $p$, then the fixed point set under $T$ is a homology $r_{0}$-sphere over $G_{p},-1 \leqq r_{0} \leqq n_{0}$. The main aim of this paper is to establish the following theorem: In the Smith Theorem, if all the integral cohomology groups of $Q$ are groups with a finite number of generators, then $T$ preserves orientation or reverses orientation according as $n_{0}-r_{0}$ is even or odd. The meaning of orientation-preserving or orientation-reversing is as follows. If $p>2$, this is given in an obvious manner. In general, in the present theorem it can be seen that $Q$ is also a homology $n_{0}$-sphere over the group of rational numbers, and hence the meaning of orientation-preserving or orientation-reversing can be settled. Moreover, for $p>2$, the two definitions will agree. The present theorem gives the positive solution of a problem proposed by Smith [Lefschetz, Algebraic topology, Amer. Math. Soc. Colloquium Publications, vol. 27, 1942, p. 373]. (Received April 23, 1951.) 